\title{
DADOS MÉDICO-LEGAIS SOBRE AFOGAMENTOS NA REGIÃO DE RIBEIRÃO PRETO (SP, BRASIL): UM PASSO PARA A PREVENÇÃO
}

\author{
MEDICO-LEGAL DATA ON DROWNING IN RIBEIRÃO PRETO REGION (SP, BRAZIL): \\ A STEP FOR PREVENTION
}

Rodrigo T. Araújo1, Carmen C. S. Martin², Bruno S. De Martinis², Martin P. Evison³, Marco A. Guimarães²

${ }^{1}$ Pós-Graduando, ${ }^{2}$ Docente. Centro de Medicina Legal. Departamento de Patologia da Faculdade de Medicina de Ribeirão Preto - USP. ${ }^{3}$ Forensic Science Program, University of Toronto at Mississauga.

CorrespondÊncia: Prof. Dr. Marco Aurelio Guimarães. CEMEL, Departamento de Patologia - FMRP-USP. Rua Tenente Catão Roxo, 2418, Ribeirão Preto / SP, Brazil, 140051-140. Tel: +55 (16) 3602-3358 ; Fax: +55 (16) 3602-3360. e-mail: mag @fmrp.usp.br

Araújo RT, Martin CCS, Martinis BS, Evison MP, Guimarães MA. Dados médico-legais sobre afogamentos na região de Ribeirão Preto (SP, Brasil): um passo para a prevenção. Medicina (Ribeirão Preto) 2008; 41 (1): 50-7.

RESUMO: Este é um estudo de prevalência com a primeira descrição específica de casos de afogamento em uma área não-costeira do Brasil, em Ribeirão Preto, estado de São Paulo, uma região com padrão de vida comparável ao de países desenvolvidos, com o objetivo de estabelecer medidas preventivas para este tipo específico de trauma. Metodologia: Uma análise da documentação médico-legal de 89 casos de afogamento ocorridos entre 2001 e 2004 . O padrão de mortes por afogamento foi estudado através de parâmetros como idade, sexo, características sócio-econômicas e avaliação do consumo de álcool. Resultados: um índice de 2,69 casos por 100.000 habitantes foi observado. O perfil predominante entre as vítimas de afogamento foi o do homem caucasiano em idade economicamente produtiva (15-59 anos), afogados acidentalmente em rios e represas na área rural durante o verão e o outono, sendo inviável a avaliação do consumo de álcool. Conclusão e Relevância: a observação do perfil da vítima de afogamento através dos relatórios médico-legais permitiu o desenvolvimento de um projeto piloto de sucesso na prevenção de afogamentos que pode ser expandido para outras áreas não-costeiras do Brasil, visando à redução do número de vítimas através da prevenção. As dificuldades de investigar a influência do consumo de álcool são discutidas.

Descritores: Medicina Legal. Epidemiologia. Ferimentos e Lesões. Afogamento. Brasil.

\section{1- INTRODUÇÃO}

O afogamento é um tipo de trauma específico definido como a aspiração de fluídos não-corporais causado por imersão ou submersão ${ }^{1}$. A Organização Mundial da Saúde (World Health Organization WHO) estima que, globalmente, 400.000 casos ocorram anualmente - uma taxa de 6,8 casos por 100.000 habitantes por ano $^{2}$.
Apesar de acidentes de trânsito em estradas, homicídios e suicídios serem mais freqüentes, o afogamento ainda permanece como uma causa significativa dentre as mortes não-intencionais, especialmente entre a população jovem e economicamente produtiva.

A prevenção é considerada a medida mais importante na redução das taxas de mortalidade devidas ao afogamento e outras causas externas ${ }^{3}$. Entender o padrão de afogamentos é um pré-requisito necessário 
para qualquer programa de prevenção e muitos estudos regionais são apresentados na literatura com este fim. Nos Estados Unidos ${ }^{4,5}$, assim como em alguns outros países ${ }^{6,7,8}$, as incidências mais altas são relatadas no sexo masculino. Entre crianças, o afogamento é responsável por 27 por cento das fatalidades devidas à causas externas não-intencionais ${ }^{9}$.

$\mathrm{O}$ afogamento pode ocorrer nas mais variadas circunstâncias. Os casos mais relatados ocorrem em água-doce, incluindo banheiras, piscinas públicas e particulares, lagos, rios e córregos. Por outro lado, fatalidades relacionadas à navegação e esportes aquáticos são mais comuns em áreas costeiras ${ }^{4,10,11}$.

Outros fatores podem ser associados com o afogamento, incluindo desordens mentais e/ou físicas, como a epilepsia. Contudo, o consumo de álcool parece ser o fator de risco mais importante uma vez que o mesmo reduz a capacidade de julgamento e o desempenho físico, condições estas que aumentam o risco de afogamento e podem afetar a sobrevivência no caso de ocorrer uma submersão ${ }^{12,13}$.

Um estudo dinamarquês mostrou que entre adultos, 39,5 por cento dos homens e 27 por cento das mulheres estavam sob influência do álcool quando se afogaram ${ }^{14}$. Smith e Brenner ${ }^{12}$ mostraram que pessoas com alcoolemia maior que $1 \mathrm{~g} / \mathrm{l}$ têm um risco aumentado de afogamento quando comparadas com pessoas que não beberam. Drogas outras que não o álcool, ou em associação com ele também foram implicadas em casos de afogamentos entre adultos jovens ${ }^{5,11}$. Há evidências que mostram que a redução do consumo de álcool pode prevenir fatalidades ${ }^{5,11,15}$.

Apesar da sua grande dimensão territorial e sua grande população, estudos dos padrões de afogamento no Brasil são limitados. Szpilman ${ }^{3}$, que tem os dados mais recentes, relatou que cerca de 8.000 casos de afogamento ocorrem anualmente no Brasil. Este estudo analisou principalmente afogamentos em áreas costeiras mais do que com que aqueles ocorridos em áreas interioranas e localidades rurais. Além disso, revelou pouco sobre o perfil das vítimas de afogamento.

Até 1998, devido às políticas ainda provenientes do governo ditatorial no Brasil que terminou em 1985, os dados médico-legais eram bastante restritos ${ }^{16}$. Em 1999, com o relaxamento das restrições governamentais no Estado de São Paulo permitiu um acordo técnico e científico entre a Universidade de São Paulo e a Secretaria de Segurança Pública do Estado. Este acordo levou à inauguração do Centro de Medicina Legal (CEMEL) na cidade de Ribeirão Preto, local de onde originaram-se os dados deste estudo assim como de outros que viabilizaram investigações epidemiológicas de homicídios ${ }^{17,18}$ e casos médico-legais relacionados ao consumo de álcool ${ }^{19,20}$ na região.

Este estudo tem como objetivo relatar os aspectos epidemiológicos das mortes por afogamento na região de Ribeirão Preto, entre os anos de 2001 e 2004. A observação do padrão de fatalidades pode permitir o aprimoramento de programas de prevenção de afogamentos nesta região ou outras áreas interioranas similares, tanto no Brasil como em outros países.

\section{2- MATERIAL E MÉTODOS}

\section{1- Caracterização da área de estudo}

A cidade de Ribeirão Preto é um centro regional econômico e administrativo no nordeste do Estado de São Paulo. Possui uma das maiores rendas per capita do Brasil e um padrão de vida equivalente ao de muitos países desenvolvidos. Tem uma área de 642 $\mathrm{km}^{2}$ e uma população regional de mais de 827 mil habitantes de acordo com o censo do ano $2000^{21}$. As temperaturas médias são de $25^{\circ} \mathrm{C}$ no verão e $18^{\circ} \mathrm{C}$ no inverno, podendo atingir máximas acima de $38^{\circ} \mathrm{C}$ no verão.

A cidade possui um grande número de piscinas residenciais e públicas. A região tem dois rios principais, o Mogi-Guaçu e o Pardo. Há muitos reservatórios de água nas áreas rurais de fácil acesso e rotineiramente utilizados para o nado recreacional e para a pesca.

\section{2- Amostra estudada}

O estudo envolveu uma revisão dos relatórios de exame necroscópico realizados em todos os 89 casos de afogamento realizados no CEMEL entre os anos de 2001 e 2004. Este último ano foi escolhido como limitante devido à implantação de um programa educativo de prevenção de afogamento na cidade de forma a permitir futuros dados comparativos.

\section{3- Análise dos dados}

Os seguintes parâmetros foram registrados:

I) sexo: masculino ou feminino;

II) etnia: africana (negros), caucasiana (brancos), miscigenada (mulatos e outras origens étnicas);

III) idade em anos completos;

IV) área: urbana ou rural;

V) data de ocorrência e estação do ano;

VI) presença ou ausência de registro de dosagem alcoólica no sangue. 
A etnia foi categorizada pelo médico-legista de acordo com a aparência do corpo, conforme o padrão de documentação oficial vigente no Brasil. Esta categorização foi mantida devida à dificuldade amplamente divulgada e reconhecida de classificar origem étnica no Brasil, assim como em outros países do mundo.

\section{4- Aprovação Ética}

O estudo foi previamente aprovado pelo Comitê de Ética em Pesquisa do Hospital das Clínicas de Ribeirão Preto (HCRP: 14552/2005). Os dados foram coletados de forma anônima, para preservar a identidade das vítimas.

\section{3- RESULTADOS}

\section{1- Estatística Geral}

Um total de 89 casos foram relatados a partir de uma população aproximada de 827.000 habitantes, o que gerou um índice de 2,69 casos por 100.000 habitantes por ano.

\section{1- Sexo}

Das 89 vítimas de afogamento, 81 eram do sexo masculino e $(91,01 \%)$ e oito do sexo feminino $(8,99 \%)$, numa proporção masculino: feminino de 10,12:1.

\section{2- Etnia}

Dos 89 casos, $60(67,41 \%)$ eram caucasianos, $18(20,20 \%)$ eram miscigenados e $10(11,23 \%)$ eram de origem africana. Um caso $(1,12 \%)$ não possuia registro de etnia documentado.

Quando a etnia foi comparada com a área de ocorrência do afogamento (urbana ou rural), foi observado que se miscigenados e de origem africana forem considerados em conjunto, $87,5 \%$ das mortes neste grupo ocorreram em áreas rurais enquanto $82,35 \%$ das mortes por afogamento em áreas urbanas envolveram indivíduos de origem caucasiana (Figura 1).

\section{3- Faixas etárias}

A Tabela I apresenta a distribuição de idade dentre os casos de afogamento de acordo com o sexo

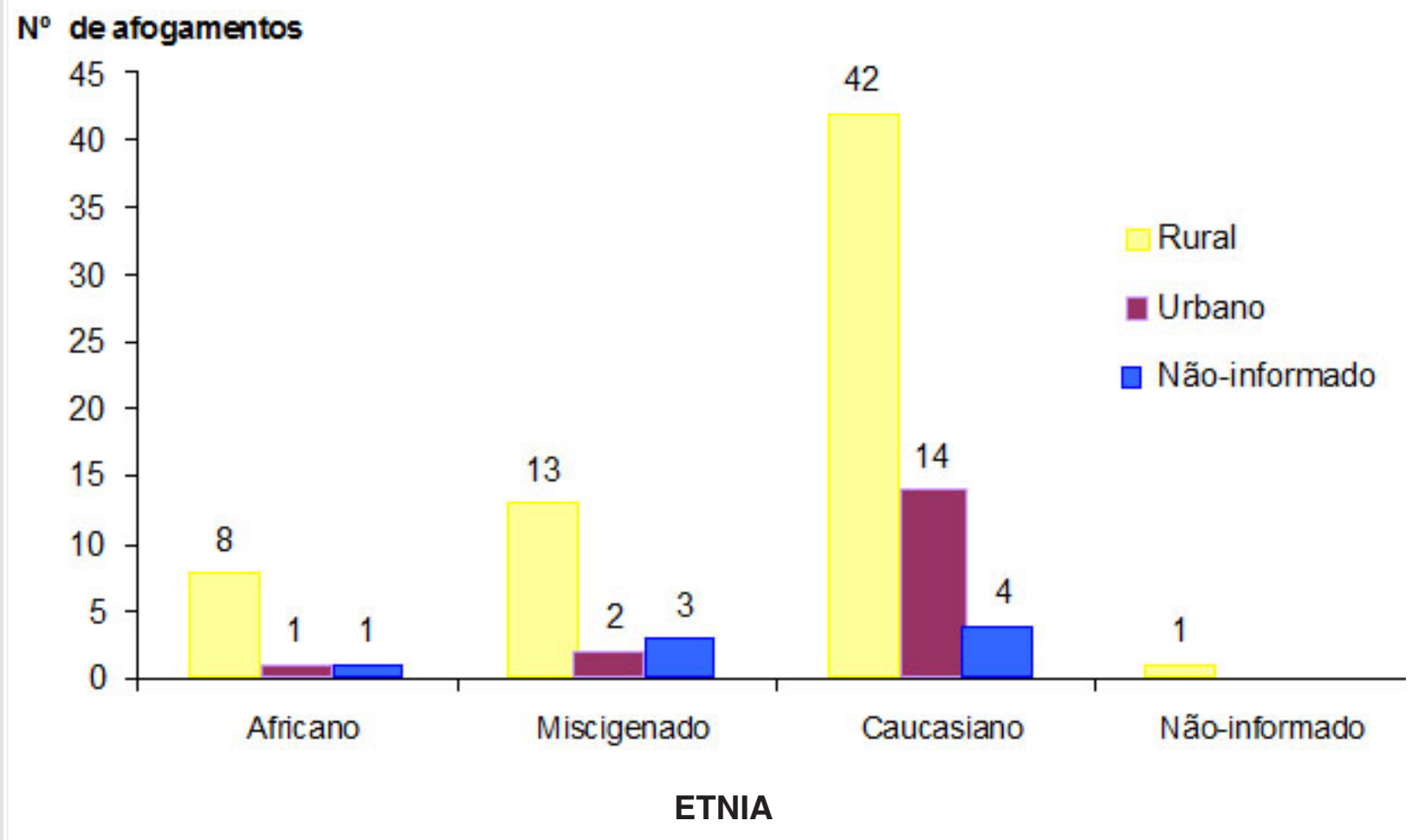

Figura 1: Distribuição dos casos de afogamento em Ribeirão Preto, Estado de São Paulo, Brasil. São apresentados o número de casos de acordo com o local de ocorrência e a etnia. 


\begin{tabular}{|c|c|c|c|c|c|c|c|c|c|c|c|c|c|}
\hline \multicolumn{14}{|c|}{ Faixa etária (em anos) } \\
\hline Etnia e Sexo & $0-4$ & $5-9$ & $10-14$ & $15-19$ & $20-29$ & $30-39$ & $40-49$ & $50-59$ & $60-69$ & $70-79$ & $\begin{array}{c}\text { Mais } \\
\text { de } 80\end{array}$ & $\begin{array}{c}\text { Sem } \\
\text { informação }\end{array}$ & Total \\
\hline Homem africano & & & 1 & & 2 & 2 & 2 & & & 1 & 1 & & $9(10,11 \%)$ \\
\hline Mulher africana & & & & 1 & & & & & & & & & $\mathbf{1}(1,12 \%)$ \\
\hline Homem miscigenado & 1 & 1 & 1 & 5 & & 4 & 2 & 3 & & & & & $17(19,1 \%)$ \\
\hline Mulher miscigenada & & & & & & & & 1 & & & & & $\mathbf{1}(1,12 \%)$ \\
\hline Homem caucasiano & 4 & 2 & 2 & 5 & 13 & 12 & 7 & 2 & 3 & 1 & & 3 & $54(60,67 \%)$ \\
\hline Mulher caucasiana & 2 & & & & & & 1 & & & & 3 & & $6(6,74 \%)$ \\
\hline Sem informação & & & & & & & & 1 & & & & & $\mathbf{1}(1,12 \%)$ \\
\hline Total & 7 & 3 & 4 & 11 & 15 & 18 & 12 & 7 & 3 & 2 & 4 & 3 & $89(100 \%)$ \\
\hline
\end{tabular}

e a etnia. O pico de incidência ocorre na faixa dos 30 aos 39 anos de idade. Este grupo é composto exclusivamente pelo sexo masculino, não tendo sido observados entre o sexo feminino na faixa dos 20 aos 39 anos de idade. Sessenta e três fatalidades $(70,79 \%)$ ocorreram entre os 15 e os 59 anos de idade, a faixa considerada mais economicamente ativa.

\section{4- Área}

Dos 89 casos, 69 ocorreram em áreas rurais $(77,53 \%)$ enquanto 15 casos $(16,85 \%)$ ocorreram em áreas urbanas. Cinco casos $(5,62 \%)$ não tiveram área de ocorrência definida nos registros. Porém, quando se considera separadamente a faixa de 1 a 5 anos de idade, $71,42 \%$ dos casos de afogamento ocorridos neste grupo foram em ambiente urbano.

\section{5- Data de ocorrência e sazonalidade}

A distribuição dos 89 casos de acordo com o ano, mês e estação do ano pode ser vista na Figura 2. A Figura 2 A mostra a distribuição anual dos casos entre 2001 e 2004, com a incidência mais alta de 29 casos (32,58\%) ocorrendo no ano de 2002.

A Figura 2B mostra o número total de casos de afogamento acumulados por mês, com a maioria de 59 casos $(66,29 \%)$ ocorrendo entre novembro e abril, quando as médias de temperatura são mais altas.

A Figura 2C mostra o acumulado de casos de afogamento pela estação do ano, com a incidência mais alta de 30 casos $(33,71 \%)$ ocorrendo durante o verão, seguido pelo outono com 25 casos $(28,09 \%)$.

$\mathrm{Na}$ Figura 2D pode ser observada a relação entre estação do ano e área de ocorrência (urbana ou rural) e notar-se a variação sazonal de afogamentos na área rural, enquanto o número de casos em áreas urbanas é pouco afetado pela estação do ano, a não ser por um pequeno decréscimo durante o inverno.

\section{6- Análise de álocool}

A alcoolemia (concentração de álcool no sangue) constava em 17 dos 89 laudos de exames necroscópicos analisados $(17,1 \%)$. Em todos os casos o exame foi realizado por cromatografia gasosa utilizando a técnica de headspace.

\section{4- DISCUSSÃO}

As fatalidades por afogamento numa taxa de 2,69 casos por 100.000 habitantes por ano observada neste estudo poderia, à princípio, ser considerada baixa em comparação com a taxa mundial de 6,8 casos por 100.000 habitantes por $a^{2} o^{2}$. Ou ainda, quando comparada com aquela obtida no único estudo brasileiro prévio a este, que relata uma taxa de 4,1 casos por 100.000 habitantes por ano, mas para uma área costeira turística no país, o estado do Rio de Janeiro ${ }^{3}$. Comparações entre estes números são difíceis, uma vez que mesmo tratando de casos de afogamento, as 


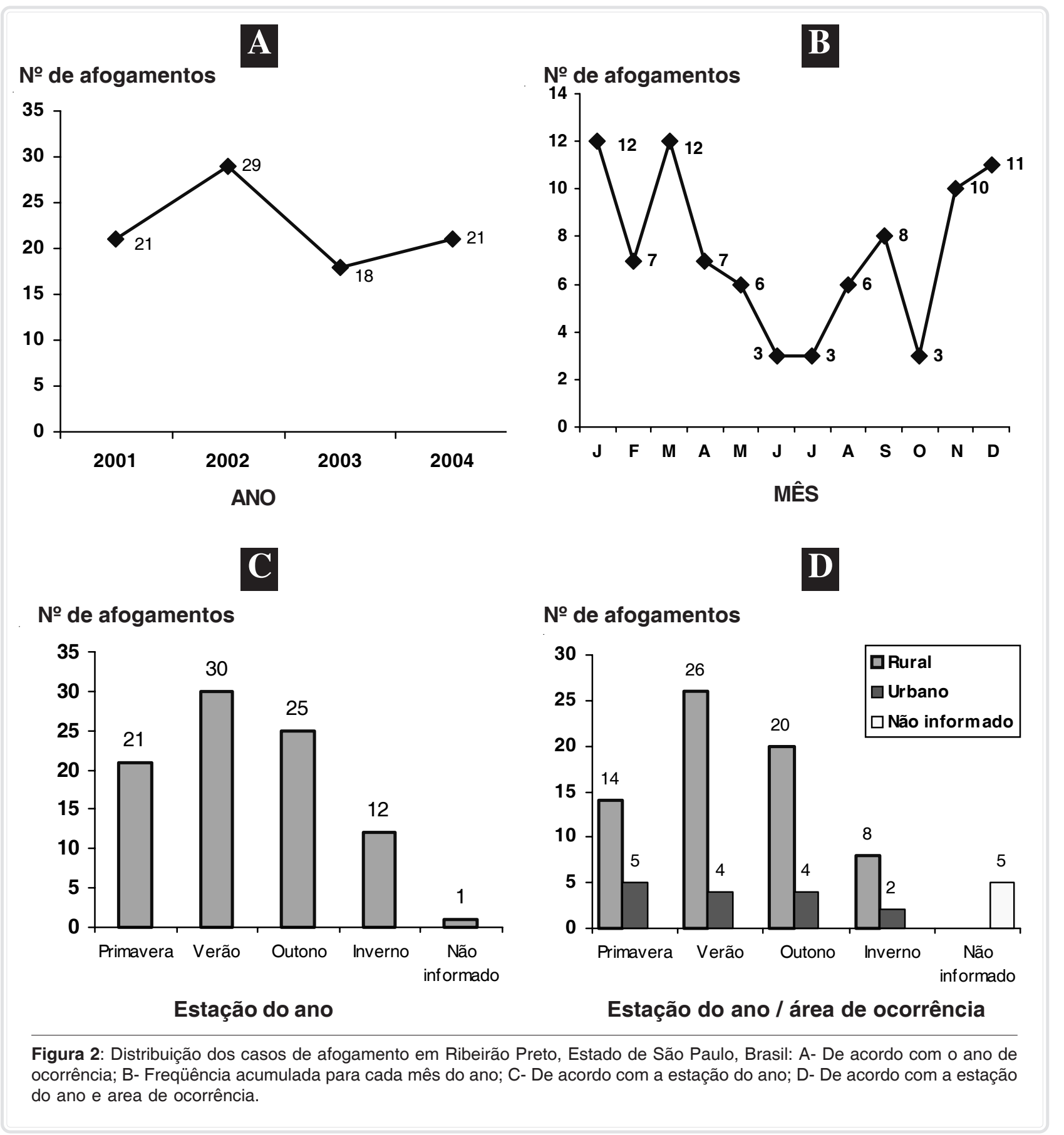

circuntâncias relacionadas para obtenção de cada taxa são distintas. Contudo, apesar dessa dificuldade, este é o primeiro dimensionamento da importância do afogamento como causa de morte em uma região não costeira do Brasil.

As observações de alguns autores ${ }^{4,10,11}$ de que fatalidades por afogamento em áreas interioranas urbanas e rurais ocorrem num padrão semelhante aos de áreas costeiras, porém com freqüências mais bai- xas, levaram à conclusão de que o afogamento em água doce (lagos, rios e córregos) não é um fenômeno isolado como freqüentemente se assume, mas um problema que se repete cronicamente e necessita de atenção específica e medidas preventivas efetivas.

É difícil, ou virtualmente impossível, para viabilizar comparações entre áreas costeiras e interioranas, calcular o percentual de cada população nas respectivas áreas que tenham exposição ao risco de afo- 
gamento. Pode-se somente supor que proporcionalmente o número de pessoas que têm acesso ao ambiente aquático numa área praiana de turismo seja muito maior que o acesso dos habitantes interioranos aos rios, represas, lagos e córregos. Assim, pode-se somente especular que a taxa de 2,69 casos por 100.000 habitantes ao ano possa ser representativa de uma gravidade maior do que a simples comparação direta com a taxa de 4,1 casos por 100.000 habitantes de áreas costeiras. De qualquer forma, a constatação dessa cifra constitui um passo importante no dimensionamento do problema.

As vítimas mais freqüentes de afogamento neste estudo foram do sexo masculino $(91,01 \%)$. Este dado é comparável aos obtidos em publicações provenientes de diferentes países encontradas na literatura 4,6,7,10 que atribuem isso à maior propensão de homens se exporem ao risco de afogamento, devido ao seu comportamento aventureiro, que inclui a natação e a pescaria em áreas não supervisionadas e ainda à associação desses fatores com o consumo de álcool.

A predominância de caucasianos $(67,41 \%)$ pode ser atribuída à sua maior frequiência na população geral no Brasil ${ }^{21}$. Entretanto, entre os casos de afogamentos urbanos a freqüência de caucasianos aumenta ainda mais (para 82,35\%) em comparação à outros grupos étnicos. Isto está possivelmente associado a fatores sócio-econômicos.

Africanos e miscigenados têm salários menores no Brasil ${ }^{22}$ e acesso reduzido à piscinas, locais de ocorrência predominante de afogamentos em áreas urbanas. Apesar de nos laudos de exame necroscópico não constar este tipo de dado (se o afogamento ocorreu em piscina, banheira, etc), informações fornecidas pelo Corpo de Bombeiros, responsável pelo resgate de corpos de vítimas de afogamento, confirmam esta hipótese.

Além disso, africanos e miscigenados utilizam mais locais não-supervisionados para natação e recreação nas áreas rurais, o que justifica que a maior parte das fatalidades desses grupos étnicos $(87,5 \%)$ ocorram nesta área.

A distribuição por faixas etárias contrasta com aquelas relatadas para os afogamentos costeiros ${ }^{3,23}$, as quais revelam pico de incidência entre 15 e 19 anos de idade e entre crianças ${ }^{2}$. Nosso estudo revelou a maior incidência ocorrendo entre os 30 e 39 anos de idade. O único achado similar na literatura foi entre homens aborígenes no Canadá ${ }^{24}$ com pico de incidência entre 25 e 34 anos de idade.
Interessante notar que a faixa etária de pico é formada exclusivamente por indivíduos do sexo masculino. Casos entre o sexo feminino estão distribuidos abaixo dos 20 e acima dos 39 anos de idade.

Apesar do aumento da participação das mulheres na economia brasileira, o aporte financeiro permanece centrado ainda entre os homens em idade produtiva em $73,3 \%$ das famílias ${ }^{25}$. O impacto social e econômico dos afogamentos em área rural, considerando-se que $70,79 \%$ deles afetam indivíduos entre 15 e 59 anos de idade, necessita de uma avaliação mais precisa para definir a dimensão real desse considerável dano social.

A maioria das fatalidades analisadas neste estudo ocorreu em áreas rurais $(77,53 \%)$. Isto pode estar relacionado à falta de supervisão e represas, lagos e rios, onde o nado recreacional e a pesca ocorrem. Em áreas urbanas, $71,42 \%$ das fatalidades ocorreram entra 1 e 5 anos de idade, fato este compatível com os dados de afogamentos entre crianças publicados pela $\mathrm{WHO}^{2}$.

Apesar dos casos de afogamento geralmente ocorrerem em meses mais quentes do ano, este padrão predomina nas áreas rurais (ver Figura 2D), onde o nado usualmente não é supervisionado, enquanto nas áreas urbanas este efeito não é tão predominante, possivelmente devido ao fato de que piscinas públicas, mais utilizadas nos meses quentes, são mais supervisionadas nestas épocas.

Dos 89 casos levantados, somente 17 continham resultados de análises de alcoolemia e nenhuma evidência de relação claramente definida com outros parâmetros deste estudo pôde ser estabelecida. As razões para o número reduzido de dosagens alcoólicas se deve ao fato de que a tecnologia para este fim (cromatografia gasosa com headspace) somente passou a ser utilizada a partir de 2002 no CEMEL. Além disso, a legislação brasileira prevê somente a obrigatoriedade da coleta de amostras de sangue em exames necroscópicos nos casos de acidentes de trânsito e não em outras formas de trauma. Assim, há necessidade de modificações de caráter legal que tornem obrigatória a coleta de sangue para análise de álcool em outras formas de trauma, incluindo o afogamento, para que estudos posteriores possam definir claramente o papel do álcool como fator interveniente.

$\mathrm{O}$ afogamento tem um impacto social e econômico significativo nas famílias atingidas devido a sua frequiência entre crianças menores e adultos economicamente produtivos. Os dados obtidos neste estudo 
podem colaborar, através do conhecimento dos fatores epidemiológicos obtidos a partir de relatórios médico-legais, na elaboração de programas de prevenção de maior efetividade para a população.

Baseado nos dados obtidos neste estudo, um projeto piloto de baixo custo para prevenção de afogamentos no qual bombeiros e salva-vidas treinaram sete policiais de uma cidade pequena na região de $\mathrm{Ri}$ beirão Preto para reconhecer os principais grupos com risco para afogamento e orientar as pessoas que costumam nadar no reservatório desta localidade. Entre 2001 e 2004 este local específico contribuiu com 6 casos do levantamento apresentado neste trabalho. Desde o estabelecimento do projeto piloto, a partir de 2004, até o momento não ocorreram mais casos de afogamento neste mesmo local.

As medidas preventivas levaram em conta não somente os fatores de risco relacionados às vítimas em potencial mas também as variáveis relacionadas ao ambiente em particular, como a sazonalidade, por exemplo.

Mais pesquisas são necessárias para investigar a influência do tipo de localidade rural sobre os afogamentos, assim como para determinar a influência real do consumo de álcool e outras drogas nestas fatalidades no Brasil. Porém, num país em desenvolvimento, isto pode levar mais tempo do que em países desenvolvidos, uma vez que os dados científicos podem estar diponíveis, mas o estabelecimento real de medidas preventivas dependem de fatores políticos e econômicos, além de mudanças culturais por parte da sociedade.

\section{5- CONCLUSÃO}

Estes são os resultados da primeira investigação sobre afogamentos em uma área não-costeira no Brasil. As limitações históricas no acesso aos dados médico-legais no Brasil causaram sérios impedimentos aos estudos de traumas e fatalidades e dificultaram, por sua vez, o entendimento dos fatores sócioeconômicos envolvidos, inviabilizando o estabelecimento de medidas preventivas efetivas para o benefício da população.

Este estudo corrobora outros que identificaram a necessidade de um entendimento específico dos fatores envolvidos no evento afogamento, que ocorre em áreas não-costeiras, não-turísticas, para a implementação de medidas preventivas apropriadas para o padrão especificamente observado nas áreas afetadas, quer seja em localidades no Brasil ou em outros países.

\section{6- AGRADECIMENTOS}

Ao Núcleo de Perícias Médico-Legais de Ribeirão Preto (NPML-RPO) e ao $9^{\circ}$ Grupamento de Bombeiros de Ribeirão Preto, pelo acesso às informações necessárias para esta pesquisa. Apoio financeiro CAPES e CNPq.

Araújo RT, Martin CCS, Martinis BS, Evison MP, Guimarães MA. Medico-legal data on drowning in Ribeirão Preto region (SP, Brazil): a step for prevention. Medicina (Ribeirão Preto) 2008; 41 (1): 50-7.

ABSTRACT: This is a prevalence study with the first specific description of drowning cases in a non-coastal area of Brazil, in the locality of Ribeirão Preto, State of São Paulo-a region with standards of living comparable to those in developed countries-regarding the establishment of preventative measures for this specific kind of injury. Methodology: An analysis of the forensic documentation of 89 drowning cases occurred between 2001 and 2004. The pattern of drowning fatalities was studied in relation to parameters like age, sex, socio-economic background and evaluation of alcohol consumption. Results: rate of 2.69 cases per 100,000 habitants per year was observed. The predominant profile among drowning victims was that of the Caucasian male of economically productive age (15 to 59 years), accidentally drowned in rivers and dams in rural areas during the summer and autumn, being impossible the evaluation of alcohol consumption. Conclusion and Relevance: the observation of the victim profile through the analysis of forensic reports allowed the development of a successful pilot program of drowning prevention that can be expanded to other non-coastal areas in Brazil, regarding the reduction of the number of victims by prevention. The difficulties in alcohol consumption analysis are discussed.

Keywords: Forensic Medicine. Epidemiology. Wounds and Injuries. Drowning. Brazil. 


\section{REFERÊNCIAS}

1 - Van Dorp JCM, Knape JTA, Bierens JJLM. Recommendations: World Congress of Drowning. Amsterdam, The Netherlands; 2002.

2 - WHO (World Health Organization) - Injury: A leading cause of the global burden of disease 2000 . Non-serial publication. WHO Press; 1999.

3 - Szpilman D, Cruz Filho F. Epidemiological profile of drowning in Brazil - 144207 deaths in 20 years study. Oral presentation. World Congress on Drowning, Amsterdam, The Netherlands; 2002.

4 - Bierens JJ, Van Deer Velde EA, Van Berkel M, Van Zanten JJ. Submersion cases in the Netherlands. Ann Emerg Med, 1989; 18(4):366-73.

5 - Mackie IJ. Patterns of drowning in Australia, 1992-1997. Med J Aust, 1999; 171: 587-90.

6 - Adams AL. The descriptive epidemiology of drowning accidents. Med J Aust, 1966; 2:1257-61.

7 - Institute of Medicine (IOM). Epidemiology of alcohol - related problems. In: Prevention and treatment of alcohol problems: research opportunities. Institute of Medicine (IOM) Washington: The National Academies Press; 1990. p. 3146.

8 - Lindholm P, Steensberg J. Epidemiology of unintentional drowning and near-drowning in Denmark in 1995. Inj Prev 2000; 6: 29-31.

9 - National Center of Injury Prevention and Control (NCIPC). Leading Causes of Death Reports, 1999-2000. Accessed in: 14/06/2007. Available from: http://webapp.cdc.gov/ sasweb/ncipc/leadcaus10.html

10 - Bremer RA, Trumble AC, Smith GS, Kessler EP, Overpeck, MD. Where Children Drown. Pediatrics 2001; 108:85-9.

11 - Quan L, Cummings P. Characteristics of drowning by different age groups. Inj Prev 2003; 9:163-8.

12 - Smith GS, Brenner RA. The changing risks of drowning for adolescents in the U.S. and effective control strategies. Adolesc Med 1995; 6:153-70.
13 - Hingson R; Howland J. Alcohol and non traffic unintended injuries. Addiction 1993; 88:877-83.

14 - Steensberg J. Epidemiology of accidental drowning in Denmark: 1989-1993 - Accid Anal Prev 1998; 30(6):77562.

15 - Fenner P. Drowning awareness: prevention and treatment. Aust Fam Physician 2000; 29:1045-9.

16 - Guimarães MA. The challenge of identifying deceased individuals in Brazil: from dictatorship to DNA analysis. Sci Justice 2003; 43:215-7.

17 - Melki JAD, Martin CCS, Guimarães MA. Assessment of methods of homicides in a Brazilian city: a preliminary study. Forensic Sci Int, 1999; 106: 19-25.

18 - Adam D. Back from the dead. Nature 2003; 423:13-4.

19 - De Martinis BS, Martin CC. Automated headspace solidphase microextraction and capillary gas chromatography analysis of ethanol in postmortem specimens. Forensic Sci Int 2002; 128(3):115-9.

20 - De Martinis BS, Ruzzene MA, Martin CCS. Determination of ethanol in human blood and urine by automated headspace solid-phase microextraction and capillary gas chromatography. Anal Chim Acta 2004; 522: 163-8.

21 - Instituto Brasileiro de Geografia e Estatística (IBGE) Accessed in: 14/06/2007. Available from: www.ibge.gov.br/ home/estatistica/populacao/censo2000.

22 - Chor D, Lima CRA. Epidemiological aspects of racial inequalities in health in Brazil. Cad Saúde Pública 2005; 21(5):1586-94.

23 - Salvamar Paulista. Accessed in: 14/06/2007. http:// www.polmil.sp.gov.br/salvamarpaulista.

24 - Weir, E. Drowning in Canada. Can Med Assoc J 2000; 162:13.

25 - Hoffmann R, Leone ET. Participação da mulher no mercado de trabalho e desigualdade da renda domiciliar per capita no Brasil: 1981-2002. Nova Economia 2004;14(2):35-58.

Recebido para publicação em 29/06/2007.

Aprovado para publicação em 13/05/2008 